\title{
A Case Report of Atypical Hyponatremia Caused by Diarrhea Following a Course of Ferrous Sulfate Supplements
}

\author{
Nasrin Dhapa ${ }^{1}$, Adithya Shekhar ${ }^{1}$, Yosof Katiby ${ }^{1}$, Krista Tenerelli ${ }^{1}$, Sampath Wijesinghe ${ }^{2}$ and Leonard Ranasinghe ${ }^{3 *}$ \\ ${ }^{1}$ Medical students, California Northstate University College of Medicine, 9700 W. Taron Dr., Elk Grove, CA 95757. \\ ${ }^{2}$ MSPA Education, Stanford School of Medicine, 1265 Welch Rd., Ste. 100, Stanford, CA, 94305-5408, USA. \\ ${ }^{3}$ Professor of Medical Education and Clerkship Director of Emergency Medicine, California Northstate University College of Medicine, 9700 W Taron
} Dr, Elk Grove, CA 95757

*Corresponding Author: Leonard Ranasinghe, Professor of California Northstate University College of Medicine, 9700 W. Taron Dr. Elk Grove, CA 95757, USA.

Received Date: December 08, 2020; Accepted Date: December 12, 2020; Published Date: January 14, 2021.

Citation: Dhapa N., Shekhar A., Katiby Y., Tenerelli K, Wijesinghe S. and Ranasinghe L. (2021) A Case Report of Atypical Hyponatremia Caused by Diarrhea Following a Course of Ferrous Sulfate Supplements. Clinical Medical Reviews and Reports, 3(1): DOI: 10.31579/2690-8794/058

Copyright: (C) 2021. Leonard Ranasinghe. This is an open-access article distributed under the terms of the Creative Commons Attribution License, which permits unrestricted use, distribution, and reproduction in any medium, provided the original author and source are credited.

\begin{abstract}
Hyponatremia, defined as a serum sodium level less than $135 \mathrm{mg} / \mathrm{mL}$, can be euvolemic, hypovolemic, or hypervolemic. Its presentation can be subtle or severe depending on whether the hyponatremia is acute or chronic. This case investigates a geriatric patient who suffered diarrhea and hyponatremia following a course of ferrous sulfate pills for anemia treatment. Given that ferrous sulfate pills usually result in constipation, this presentation was abnormal. Literature review and comparison to prior atypical situations resulted in the conclusion that the patient's diarrhea was likely a result of her Crohn's diagnosis. The subsequent resulting hyponatremia was likely due to this diarrhea coupled with her prescription thiazide diuretic which can cause hyponatremia as a common side effect. This report allows for physicians to understand potential causes behind atypical electrolyte abnormalities in their patients and hence, find an effective treatment plan.
\end{abstract}

Keywords: diarrhea, hyponatremia, ferrous sulfate, thiazide, anemia, geriatric

\section{Abbreviations}

GI: gastrointestinal;

SIADH- Syndrome of inappropriate antidiuretic hormone secretion;

UTI- Urinary tract infection;

IBD- Inflammatory Bowel Disease;

IV- Intravenous

\section{Introduction}

Iron deficiency anemia has persisted as the most common cause of anemia in the world for many years [1]. In America, about 5\% of women and 2\% of men are diagnosed with it. Within the elderly population, iron deficiency anemia becomes increasingly prevalent after age 80 , reaching rates of up to $50-60 \%$ in nursing homes [1]. Meanwhile, many older individuals develop chronic inflammation, which alters iron metabolism and can further complicate measuring iron status. Concerning adverse effects include increased risk of chronic diseases including diabetes, heart disease, and colorectal cancer [2]. Iron absorption may be limited by abnormal intestinal morphology and clinical manifestations associated with heart failure and diabetes related gastroparesis [3].

The most common treatment prescribed for iron deficiency anemia is ferrous sulfate, the better absorbed of the two forms of iron supplements that are primarily used [1]. The daily dosage recommended is $150 \mathrm{mg} / \mathrm{day}$ to $180 \mathrm{mg} /$ day divided into two to three doses a day. Within a week, reticulocyte counts begin to increase, and within three weeks, hemoglobin numbers also begin to rise. Considering these quick results, these supplements are desirable given they are relatively safe, effective, and inexpensive to treat iron deficiency anemia. However, the main side effect patients run into when taking these supplements are gastrointestinal issues including malabsorption. In patients with complications, such as lesions to the gastrointestinal (GI) tract, it can be even more difficult to control anemia because the patient's GI blood loss can cause greater loss in iron stores than that which they can absorb [4].

It is important to understand the reason behind these GI symptoms in patients taking oral iron supplements. Oral ferrous sulfate results in oxidation of iron, leading to damage in the esophageal tract and the stomach. A study was done using 1,300 gastrointestinal tract biopsies from thirty-three patients. From this, $0.9 \%$ of the biopsies showed crystalline iron deposits. Damage to the tract resulting from iron medication was present in $0.7 \%$ of biopsies. Researchers concluded that iron ingestion can induce and worsen erosion to the mucosa in the GI tract. Histological analysis showed ulcers in the mucosa in the regions where there was extracellular iron particle deposition. Evidently, the presence of iron deposits causes damage within the intestinal lining, leading to the noted gastrointestinal symptoms experienced in patients taking iron supplements [5]. 
Hyponatremia is one of the most common electrolyte abnormalities found in medical settings. It can be defined as a serum sodium concentration of $<135 \mathrm{meq} / \mathrm{l}$. The etiology is multifactorial and may be euvolemic, hypovolemic, or hypervolemic, resulting from a dysfunction in the homeostasis of water and sodium [6]. Water intoxication, the excess intake of water, can dilute the concentration of sodium in the body leading to dilutional hyponatremia. This form of hyponatremia is categorized as hypervolemic hyponatremia given the large increase in antidiuretic hormone which promotes water reabsorption in the kidneys. Similarly, hypovolemic hyponatremia may arise in patients with excessive loss of sodium from excessive sweating, vomiting, or decreased sodium reabsorption in the kidneys [6]. Euvolemic hyponatremia can be caused by the syndrome of inappropriate antidiuretic hormone secretion (SIADH) which is typically diagnosed by following a set criterion and is only a diagnosis of exclusion rather than a primary differential to go by. The etiology of SIADH is varied and can manifest from the central nervous system, malignancies, drugs, surgery, pulmonary disease, HIV, hereditary causes, polydipsia, low sodium intake, exercise or can even be idiopathic in nature [7]. The medications that can induce SIADH are also varied and include anti-epileptics, anti-cancer, anti-psychotics, antiinflammatory medications, and exogenous hormones [8].

Acute symptoms of hyponatremia are characterized by neurological symptoms such as cerebral edema, seizures, and coma and can manifest in less than 2 days with increased neurological symptoms caused by brain swelling due to water moving via osmosis [6]. Chronic hyponatremia symptoms, characterized by an onset lasting more than 48 hours, include nausea, vomiting, lethargy, postural hypotension, and ataxia [6]. Chronic hyponatremia is typically less severe due to the ability of the body to adapt in order to protect the brain. Gastrointestinal symptoms are typically seen in chronic patients, however some neurologic symptoms such as gait disturbances and falls can persist, especially in the elderly [6].

Treatment for hyponatremia varies and is dependent on duration, symptoms, and etiology of the condition. In most cases, patients should be treated by discontinuing the culprit medication and following up with fluid administration. Acute hyponatremia is generally treated with rapid infusion of $5 \% \mathrm{NaCl}$ or $8.4 \% \mathrm{NaCO} 3$ to provide urgent correction limiting severe cerebral defects and herniation [9]. As chronic hyponatremia tends to present with milder symptoms, treatment protocols depend on the symptoms present but include fluid restriction and vasopressin [7].

This case will investigate an eighty-three old female presenting to the clinic with a chief complaint of feeling tired. Patient has been prescribed and is actively taking ferrous sulfate due to a diagnosis of anemia. However, she has been experiencing diarrhea following these supplements which she understands is uncommon. Patient does have a history of hypertension for which she takes hydrochlorothiazide. At her last visit with her primary care physician, the complete metabolic panel results showed low levels of sodium and chloride. Patient confirms she has been feeling extremely tired the last few days and is also slightly confused as noticed by the PCP. To repeat labs and receive quick results, PCP suggested the patient make a visit to the ED. On physical exam, the patient has a heart rate of $73 \mathrm{bpm}$, respiration rate of 20 breaths/min and a blood pressure reading of $134 / 67 \mathrm{mmHg}$. O2 saturation is sufficient on room air at $97 \%$. Patient has a Body Mass Index (BMI) of $20.11 \mathrm{~kg} / \mathrm{m}^{2}$. Past medical history of the patient is significant for Crohn's disease, gastroesophageal reflux disease (GERD), osteoarthritis, recurrent urinary tract infection (UTI), and arrhythmia. Her current medications include allegro, aspirin, atenolol, ferrous sulfate, chondroitin-glucosamine, Flonase nasal spray, hydralazine, hydrochlorothiazide, multivitamin, omega-3 fish oil, pantoprazole, vitamin D3, and vitamin E. Repeated lab results in the ED demonstrate that sodium level is $126 \mathrm{mmol} / \mathrm{L}$ and chloride level is $86 \mathrm{mmol} / \mathrm{L}$, both of which are remarkably low resulting in a diagnosis of hyponatremia and hypochloremia. This report will investigate possible causes behind the diarrhea and subsequent atypical hyponatremia experienced by this patient.

\section{Materials and Methods}

In order to conduct a successful case study, we have used a qualitative approach to investigate a collection of cases in order to assess the above case. Since the elderly population is estimated to double within the next 30 years, it is important to highlight the physiological changes in elderly patients and atypical clinical presentations that can accompany.

It was also critical to investigate the usage of iron supplements and common side effects such as constipation along with some abnormal side effects. By looking into cases that had atypical hyponatremia, we then focused our review on finding articles that would provide us with information on our specific patient's situation. We also used sources from geriatric studies to understand the mechanism of iron metabolism in the elderly, and subsequent risks associated with irregular iron levels in these patients.

In this case report, we initially used scholarly scientific sources to evaluate the physiology of hyponatremia. This included investigating different causes such as the use of diuretics, inappropriate antidiuretic hormone release, and endocrine abnormalities [10]. Before heading into the analysis of our patient's case it was critical to understand the physiology and methods behind hyponatremia. Once this was solidified, we investigated real-world situations to understand prior reasonings behind geriatric hyponatremia in the emergency department. As our patient is eighty-three years old, we primarily focused on geriatric cases to highlight side effects from pharmaceuticals, like hyponatremia in elderly patients using hydrochlorothiazide or other thiazides [11].

\section{Results and Discussion}

An interesting case by Lwanga et al. (2017) investigated an eighty-sixyear-old woman who presented with serum sodium levels of $99 \mathrm{mEg} / \mathrm{L}$, diagnosing her with hyponatremia. Initial complaints included difficulty walking. The patient was also found to be twenty-four pounds below her previous weight, making her underweight. Additionally, her blood pressure of 233/122 $\mathrm{mmHg}$ classified her as having severe hypertension. After disproving other known causes of hyponatremia, the patient was found to have SIADH or a potential adverse reaction to a diuretic. Given that this patient is taking a thiazide medication it is highly likely that she is suffering from a thiazide associated hyponatremia. The nature of such hyponatremia is that it can occur within a few days or as late as a few years after taking the medication. Risk factors include older age, low BMI, and female gender. In this patient, management included administration of $3 \%$ sodium chloride [12]

This prior case report demonstrates that consumption of a thiazide medication can lead to hyponatremia. All other risk factors that were identified, including low weight, female sex, and older age are all present in our patient as well [11]. Thus, it is possible that the hyponatremia in our patient is amplified by the usage of the medication that she is currently taking for hypertension. As mentioned earlier, it is uncommon for iron supplements to result in diarrhea and rather are more associated with constipation. In our case, although the patient is experiencing diarrhea as a result of iron pills and this may be the root cause behind her hyponatremia, the significantly low levels of sodium ions could be a combination of this diarrhea and consumption of thiazide medication.

Given the history of thiazide use and volume depletion, our patient may have diuretic induced hyponatremia. Long-term usage of thiazide medication coupled with diarrhea due to iron supplementation may have exacerbated our patient's hypovolemic state. Thiazide induced hyponatremia occurs due to an increase in water intake (polydipsia induction) and lowering of dilution ability/water excretion of the kidneys. 
Sodium and potassium concentrations are higher in the urine than in the plasma and therefore result in a drop in osmolarity of sodium in the plasma. Thiazide induced hyponatremia is usually a chronic condition, and therefore should be corrected slowly. Potassium should be administered as well to prevent arrhythmia.

Our patient has a history of Crohn's disease and UTI presenting with diarrhea. Crohn's disease, alongside iron supplementation, can also result in diarrhea. Iron supplementation with Crohn's disease is poorly tolerated as higher doses of oral iron have been shown to increase risk of GI side effects in irritable bowel disease (IBD) patients [12]. The duodenum, the primary site for iron absorption, is damaged in Crohn's disease. Therefore, oral absorption is limited, preventing stabilization of the anemia and allowing for more gastrointestinal side effects to occur. The increase in side effects is due to the prevalence of free radical generation of colonic iron [13]. These free radicals damage the mucosa and lumen of the gut, impairing the integrity of the gut wall and promoting carcinogenesis. In addition to the damage to the gut mucosa, the gut microbiome is significantly affected. In a study comparing oral and IV iron supplementation, it was shown that in IBD patients the microbiome was significantly altered in oral iron supplementation compared to IV administration [14].

In a meta-analysis of randomized controlled studies, oral ferrous sulfate was found to cause significant GI distress compared to placebos or IV iron treatment. Of the reported GI distress, $8 \%$ of patients reported diarrhea [15]. The loss of fluid due to the diarrhea alongside the hyponatremia suggests a hypovolemic cause. However, the severity of the hyponatremia does suggest that the diarrhea is not the only cause, as it has not been reported as a significant loss of fluid. A secondary mechanism must be at play to warrant such a drop in sodium.

Thiazide associated hyponatremia is a well-documented side effect of thiazide use. The mechanism of action of thiazide necessitates fluid as well as sodium loss through the urine at the distal convoluted tubule site of the nephron. The severity of the condition varies but is most common in elderly females soon after thiazide use. Therefore, hypovolemic hyponatremia is the most common manifestation of the condition, as fluid and sodium loss lead to a lower osmolarity.

\section{Conclusion}

As established before, the patient's diarrhea was most likely initially a result of the oral iron supplementation alongside the patients Crohn's disease. The severity of the hyponatremia however cannot be accounted for by just a recent bout of diarrhea, rather a secondary process must be occurring. The thiazide medication that the patient was prescribed for her hypertension is yet another known cause of hyponatremia, especially in elderly females. Thiazides cause a loss of sodium and water to lower blood pressure. Her rare case of diarrhea alongside oral iron supplementation and thiazide induced hyponatremia combine to form a toxic combination that likely caused our patient's sodium level to drop to $126 \mathrm{mmol} / \mathrm{L}$. Our case exhibits the rare complications of oral iron supplementation in elderly patients who are receiving treatment for hypertension as well as suffering from gastrointestinal illnesses like Crohn's Disease. It is not only important to be aware of complications when treating anemia and hypertension but to be proactive in intervention to prevent such scenarios. Hence, physicians must be aware of the combined side effects of the medication they give, as well as the route in which they administer them.

\section{Acknowledgements}

Sampath Wijesinghe, DHSc. - Reviewer of Final Manuscript.

\section{Conflict of Interest}

The authors declare that there are no conflicts of interest.

\section{References}

1. Camaschella, C. (2019). Iron Deficiency. Blood.

2. Fairweather-Tait SJ, Wawer AA, Gillings R, Jennings A, Myint PK. (2014) Iron status in the elderly. Mech Ageing Dev. 136137(100):22-28.

3. Palleschi, L., \& Nunziata, E. (2017). Iron deficiency and iron deficiency anaemia in elderly patients with chronic heart failure. Geriatric Care, 3(4).

4. Johnson-Wimbley, T. D., \& Graham, D. Y. (2011). Diagnosis and management of iron deficiency anemia in the 21 st century. Therapeutic advances in gastroenterology, 4(3), 177-184.

5. Sunkara, T., Caughey, M. E., Nigar, S., Olivo, R., \& Gaduputi, V. (2017). Iron Pill Gastritis: An Under Diagnosed Condition with Potentially Serious Outcomes. Gastroenterology research, 10(2), 138-140.

6. Sahay, M., \& Sahay, R. (2014). Hyponatremia: A practical approach. Indian journal of endocrinology and metabolism, 18(6), 760-771.

7. Sterns, R. (2018, September 11). Causes of hypotonic hyponatremia in adults. Retrieved March 2, 2020.

8. Gross P. (2012) Clinical management of SIADH. Therapeutic Adv Endocrinol Metabolism. 3(2):61-73.

9. Ghanem, A. N. (2018). Therapy of Hyponatremia: End of Era or Minority Report? Biomedical Journal of Scientific \& Technical Research, 11(4).

10. Schlanger LE, Bailey JL, Sands JM. (2010) Electrolytes are aging. Adv Chronic Kidney Dis. 17(4):308-319.

11. Filippatos TD, Makri A, Elisaf MS, Liamis G. (2017) Hyponatremia in the elderly: challenges and solutions. Clin Interv Aging. 12:1957-1965.

12. Lwanga, A., Mohamed, A., Patel, M., \& Serrano, A. (2017). Severe Hyponatremia Presenting with Minimal Symptoms. Cureus, 9(11), e1830.

13. Carla J. Gargallo-Puyuelo, Erika Alfambra, Jose Antonio García-Erce, Fernando Gomollon (2018). Iron Treatment May Be Difficult in Inflammatory Diseases: Inflammatory Bowel Disease as a Paradigm. Nutrients MDPI.

14. Thomas Lee, Thomas Clavel, Kirill Smirnov, Annemarie Schmidt, Ilias Lagkouvardos, Alesia Walker, Marianna Lucio, Bernhard Michalke, Philippe Schmitt-Kopplin, Richard Fedorak, and Dirk Haller (2017). Oral versus intravenous iron replacement therapy distinctly alters the gut microbiota and metabolome in patients with IBD.

15. Tolkien Z, Stecher L, Mander AP, Pereira DI, Powell JJ. Ferrous sulfate supplementation causes significant gastrointestinal sideeffects in adults: a systematic review and meta-analysis. PLoS One. 2015;10(2):e0117383. Published 2015 Feb 20. 
This work is licensed under Creative Commons Attribution 4.0 License

To Submit Your Article Click Here: Submit Manuscript

DOI: $10.31579 / 2690-8794 / 058$
Ready to submit your research? Choose Auctores and benefit from:

* fast, convenient online submission

* rigorous peer review by experienced research in your field

* rapid publication on acceptance

* authors retain copyrights

* unique DOI for all articles

* immediate, unrestricted online access

At Auctores, research is always in progress.

Learn more www.auctoresonline.org/journals/international-journal-ofclinical-case-reports-and-reviews- 